\title{
Concordância Interobservador no Diagnóstico Citológico de Atipia Escamosa de Significado Indeterminado Favorecendo Lesão de Alto Grau e de Lesão Intraepitelial Escamosa de Alto Grau nas Lesões do Colo Uterino
}

doi: https://doi.org/10.32635/2176-9745.RBC.2022v68n1.1338

\author{
Interobserver Agreement in Cytological Diagnosis of Atypical Squamous Cell, Cannot Exclude a High-Grade Lesion and High- \\ -Grade Squamous Intraepithelial Lesion in Cervical Lesions \\ Concordancia Interobservador en el Diagnóstico Citológico de Atipia Escamosa de Significado Indeterminado Favoreciendo \\ Lesión de Alto Grado y Lesión Intraepitelial Escamosa de Alto Grado en Lesiones del Cuello Uterino
}

Leopoldo Silocchi Pergo'; Cibele Feroldi Maffini²; Rita Maira Zanine³; Luiz Martins Collaço4; Ana Paula Martins Sebastião5

RESUMO

Introduçáo: $\mathrm{O}$ exame de Papanicolau é uma importante ferramenta na triagem do carcinoma do colo uterino. O diagnóstico citológico de atipias celulares escamosas de significado indeterminado favorecendo lesão de alto grau (ASC-H) é a categoria de menor concordância interobservador. Objetivo: Avaliar o grau de concordância interobservador para os diagnósticos de ASC-H e de lesôes intraepiteliais escamosas de alto grau (LIEAG) em um hospital terciário e avaliar a capacidade do diagnóstico de ASC-H para predizer lesóes de maior grau. Método: Foram coletadas lâminas de pacientes atendidas entre 2007 e 2015 no Serviço de Anatomia Patológica do hospital, com diagnósticos originais de ASC-H ou LIEAG realizados pelo mesmo patologista, colposcopia e biópsia, quando indicadas, pelo mesmo ginecologista. Essas citologias foram posteriormente revisadas por outros dois patologistas separadamente e às cegas. Ambos tiveram acesso a dados sobre idade no momento do diagnóstico para reproduzir o diagnóstico da prática clínica. Resultados: Houve 65,1\% de lâminas listadas com ASC-H e 34,9\% com LIEAG. As duas revisôes concordaram concomitantemente com o diagnóstico original em 54,7\%. Os índices kappa para os dois diagnósticos e somente para ASC-H foram, respectivamente, 0,46 e 0,49 (concordâncias moderadas). Das lâminas originalmente interpretadas como ASC-H, 68,3\% resultaram em lesões de maior grau na histologia. Conclusão: Os dados mostraram uma concordância moderada entre os patologistas para o diagnóstico de ASC-H. É importante destacar que o diagnóstico de ASC-H correspondeu à lesão de maior grau de malignidade na histologia, demonstrando que essas lesóes devem ser seguidas clinicamente como LIEAG.

Palavras-chave: neoplasias do colo do útero; variações dependentes do observador; teste de Papanicolau; lesóes intraepiteliais escamosas.

\section{ABSTRACT}

Introduction: The Papanicolaou test is an important screening exam for cervical carcinoma. The cytological diagnosis of undetermined atypical squamous cells favoring high-grade lesion (ASC-H) is the category with the least interobserver concurrence. Objective: Evaluate the interobserver concurrence for the ASC-H and high-grade squamous intraepithelial lesions (HSIL) categories at a teaching hospital and to estimate ASC-H's capacity to predict higher grade lesions. Method: Smears from patients admitted from 2007 to 2015 whose original diagnosis was made by one pathologist, in addition to colposcopy and biopsy, when indicated, made by one gynecologist were collected in the Pathologic Anatomy Service of the hospital. The cytology was reviewed by two other pathologists separately and blindly. Both reviewers had access to data about age at the moment of the diagnosis in order to reproduce the clinical diagnosis. Results: There were $65.1 \%$ smears considered as ASC-H and $34.9 \%$, as HSIL. The reviews concurred simultaneously with the original diagnosis in $54.7 \%$ of the cases. The kappa indexes for both categories and only for ASC-H were, respectively, 0.46 and 0.49 (moderate concurrence). $68.3 \%$ of the smears primarily described as ASC-H resulted in higher grade lesions in histology. Conclusion: The data showed a moderate concurrence between the pathologists for the ASC-H's diagnosis. It is important to highlight that ASC-H matched with higher grade lesions at the histology, needing follow-up as HSIL.

Key words: uterine cervical neoplasms; observer variation; Papanicolaou test; squamous intraepithelial lesions.

\section{RESUMEN}

Introducción: La prueba de Papanicolaou es un importante examen de detección del carcinoma del cuello uterino. El diagnostico citológico de las células escamosas atípicas, no se descarta una lesión de grado alto (ASC-H) es la categoría de menor acuerdo interobservador. Objetivo: Los objetivos de este estudio fueron evaluar el grado de concordancia interobservador para los diagnósticos de atipias escamosas de significado indeterminado favoreciendo lesión de alto grado (ASC-H) y de lesiones intraepiteliales escamosas de alto grado (LIEAG) en un hospital terciario de Curitiba (PR) y evaluar la capacidad del diagnóstico de ASC-H de predecir las lesiones de mayor grado. Método: Se recogieron del Servicio de Anatomía Patológica del hospital las láminas de pacientes atendidas entre 2007 y 2015, con diagnósticos originales de ASC-H o LIEAG realizados por el mismo patólogo y colposcopia y biopsia, cuando indicadas, por el mismo ginecólogo. Esas citologías fueron revisadas después por otros dos patólogos separadamente y a ciegas. Ambos tuvieron acceso a datos sobre edad en el momento del diagnóstico para reproducir el diagnóstico de la práctica clínica. Resultados: Hubo el $65,1 \%$ de las láminas señaladas con ASC-H y el $34,9 \%$, con LIEAG. Las revisiones concordaron concomitantemente con el diagnóstico original en el 54,7\%. Los índices kappa para los dos diagnósticos y solamente para ASC-H fueron, respectivamente, 0,46 y 0,49 (concordancias moderadas). De las láminas originalmente interpretadas como ASC-H, 68,3\% resultaron en lesiones de mayor grado en la histología. Conclusión: Hubo una concordancia moderada entre los patólogos para la categoría ASC-H. Se destaca también la correspondencia de ASC-H con lesiones de mayor grado en la histología, lo que dirige su seguimiento clínico como LIEAG. Palabras clave: neoplasias del cuello uterino; variaciones dependientes del observador; prueba de Papanicolaou; lesiones intraepiteliales escamosas.

\footnotetext{
1-5Universidade Federal do Paraná, Setor de Ciências da Saúde, Complexo Hospital de Clínicas. Curitiba (PR), Brasil. E-mails: leopoldosilocchi@gmail.com; cimaffini@gmail.com ritazanine@yahoo.com.br; Imcollaco@uol.com.br; anapms@ufpr.br. Orcid iD: https://orcid.org/0000-0002-4070-389X; Orcid iD: https://orcid.org/0000-0001-87157470; Orcid iD: https://orcid.org/0000-0001-9210-2925; Orcid iD: https://orcid.org/0000-0002-3215-1650; Orcid iD: https://orcid.org/0000-0002-2993-577X

Endereço para correspondência: Leopoldo Silocchi Pergo. Rua Itupava, 72, apto. 83 - Alto da Rua XV. Curitiba (PR), Brasil. CEP 80045-140. E-mail: leopoldosilocchi@gmail.com
} 


\section{INTRODUÇÃO}

O carcinoma do colo uterino é causado pela infecção persistente de alguns grupos oncogênicos do Papilomavírus Humano (HPV) e representa um dos tumores mais frequentes na população feminina e uma das principais causas de morte em mulheres por câncer no Brasil ${ }^{1}$. A infecção pelo HPV é a doença sexualmente transmissível (DST) mais comum em todo o mundo e grande parte das pessoas sexualmente ativas terá contato com o vírus em algum momento da vida $^{2}$. Para cada ano do triênio 2020-2022, o Instituto Nacional de Câncer José Alencar Gomes da Silva estimou 16.710 casos no Brasil ${ }^{1}$. Em virtude disso, são importantes as ferramentas de trabalho que rastreiam lesôes precursoras, permitindo intervençóes precoces à evolução do tumor em questão.

Nesse sentido, o exame citológico do colo do útero (exame de Papanicolau) consiste em instrumento para a triagem de mulheres que estáo expostas aos riscos de desenvolver o carcinoma cervical. No Brasil, o Ministério da Saúde recomenda iniciar a triagem aos 25 anos ou três anos após a primeira atividade sexual, refazendo o exame uma vez ao ano nos dois primeiros anos e trienalmente nos anos que se seguem. Após os 65 anos, caso os dois últimos exames tenham sido normais, o rastreamento pode ser finalizado ${ }^{3}$.

O exame de Papanicolau possui, contudo, algumas particularidades que podem dificultar a verdadeira detecção desse carcinoma. Esses entraves levam ao subtratamento de mulheres com lesôes potencialmente agressivas e também ao sobretratamento daquelas com lesôes limítrofes ou de baixo grau para malignidade, resultando em danos psicológicos a mulheres e aumento nos gastos públicos em saúdé ${ }^{4}$ Um exemplo disso é a baixa concordância diagnóstica entre os patologistas na categoria citológica de células escamosas atípicas de significado indeterminado quando náo se pode excluir lesão de alto grau (ASC-H).

Pesquisas apontam que o grau de concordância diagnóstica para ASC-H é o menor entre todas as categorias diagnósticas do exame citológico ${ }^{5-7}$. Em 2001, o estudo The Bethesda Interobserver Reproducibility Study (BIRST) ${ }^{6}$ identificou que a terminologia ASC-H apresentava a pior reprodutibilidade $(22,4 \%)$ entre as categorias do Sistema de Bethesda. Em 2014, a mesma metodologia foi utilizada para conduzir o BIRST- $2^{7}$, no qual a concordância diagnóstica interobservador para ASC-H mais do que dobrou (60\%). Com isso, fica evidente a importância dos citopatologistas melhorarem a utilização do Sistema de Bethesda para alcançar um maior consenso na interpretaçáo de citologias cervicais.
Sendo assim, o presente estudo teve como objetivo avaliar, de forma retrospectiva e observacional, o grau de concordância interobservador para o diagnóstico de ASC-H e de lesão intraepitelial escamosa de alto grau (LIEAG) (neste caso, quando houve algum resultado limítrofe no seguimento) entre citopatologistas do Complexo do Hospital de Clínicas da Universidade Federal do Paraná (CHC-UFPR). Além disso, foi avaliada a capacidade do diagnóstico de ASC-H de predizer lesóes de maior grau de malignidade no exame histológico.

\section{MÉTODO}

Este trabalho foi aprovado pelo Comitê de Ética em Pesquisa em Seres Humanos do CHC-UFPR, sendo registrado sob o número CAAE: 96028318.1.0000.0096. Com informaçóes obtidas do banco de dados do Serviço de Anatomia Patológica (SAP) do CHC-UFPR, esta pesquisa não precisou da aplicação de um Termo de Consentimento Livre e Esclarecido (TCLE).

Trata-se de estudo analítico, observacional, de corte transversal e com coleta de dados retrospectiva.

Foram recuperadas dos arquivos do SAP do CHC-UFPR as lâminas de exames preventivos de carcinoma do colo uterino com diagnóstico de ASC-H ou LIEAG de pacientes atendidas no Ambulatório de Tocoginecologia do hospital entre janeiro de 2007 e dezembro de 2015. Os esfregaços com diagnóstico de LIEAG inclusos são aqueles cujas pacientes foram diagnosticadas, em algum momento do seguimento, como ASC-H. Nesses casos, a primeira colposcopia após o diagnóstico de ASC-H não evidenciou achados anormais e a junção escamocolunar (JEC) não era visível ou parcialmente visível, sendo necessário investigar o canal endocervical. Uma vez que o resultado desse exame fora negativo, indicaram-se novas citologia e colposcopia em seis meses, momento no qual o diagnóstico de LIEAG foi feito. Tanto as lâminas com diagnóstico de ASC-H quanto de LIEAG foram originalmente avaliadas pelo mesmo médico-patologista do serviço, cujos diagnósticos, neste estudo, foram designados como "avaliação original".

Foram excluídas do estudo as lâminas de pacientes cujos dados clínicos não foram identificados no banco de dados do Ambulatório de Tocoginecologia ou do SAP do CHC-UFPR e também as lâminas que se encontravam deterioradas ou descoradas, impossibilitando sua correta revisão.

Dois citopatologistas especialistas do Departamento de Patologia Médica do CHC-UFPR realizaram as revisóes das lâminas. O primeiro revisor é membro da Academia Internacional de Citopatologia (IAC) e seus diagnósticos foram referidos no texto como "primeira revisão". O segundo revisor é filiado da IAC e seus diagnósticos foram referidos como "segunda revisão". 
A revisão dos casos foi realizada pelos dois citopatologistas de forma independente e às cegas, isto é, sem conhecimento do diagnóstico citológico da "avaliação original" ou do histológico obtido a partir de conização a frio. Ambos os revisores tiveram acesso às informaçóes clínicas sobre idade no momento do diagnóstico e estado de menopausa, quando disponíveis, com o objetivo de reproduzir a rotina da prática clínica. Esses casos foram analisados de acordo com a terminologia recomendada pelo Sistema de Bethesda ${ }^{6}$ e conforme a Nomenclatura Brasileira para Laudos Citopatológicos em uso atual pelo SAP do hospital, na qual a categoria NIC I (neoplasia intraepitelial cervical grau I) foi referida como lesão intraepitelial escamosa de baixo grau (LIEBG) e as terminologias NIC II e NIC III (neoplasias intraepiteliais cervicais grau II e grau III), como LIEAG.

Os exames colposcópicos das pacientes com diagnóstico de ASC-H e de LIEAG incluídas neste estudo foram feitos por médicos-residentes em ginecologia e em patologia cervical, sob supervisão de um único colposcopista experiente e responsável pelo serviço. Foram excluídas as amostras cujo resultado colposcópico náo tenha sido supervisionado pelo colposcopista mencionado, pois esse desfecho poderia representar um diagnóstico errado.

Para as colposcopias negativas, quando não se identificou a área alterada para ser biopsiada, a paciente foi seguida com citologia oncótica em seis meses, sendo este o resultado considerado como "Desfecho" nesses casos. As pacientes biopsiadas e com histologia negativa ou de LIEBG receberam alta e foram encaminhadas à Unidade Básica de Saúde (UBS); para essas pacientes, foi considerado como "Desfecho" o resultado da biópsia colpoguiada. Já as pacientes com LIEAG na biópsia foram submetidas à conização a frio. Os diagnósticos histológicos de "Desfecho" referentes às biópsias e às conizaçôes a frio foram adquiridos a partir do banco de dados do SAP do CHC-UFPR.

A análise epidemiológica quanto à idade do diagnóstico foi efetuada a partir das informaçóes do banco de dados do SAP do CHC-UFPR.

Para descrição da variável quantitativa contínua "idade", foram usados média, valores mínimo e máximo e desvio-padrão (DP), ao passo que, para sumarização das variáveis qualitativas categóricas ("diagnósticos citológicos da primeira revisão", "diagnósticos citológicos da segunda revisão", "diagnósticos citológicos da avaliação original" e "diagnósticos histológicos do Desfecho"), apontaram-se frequências e percentuais. Para comparação das revisôes quanto à probabilidade de concordância com a avaliação original, foi considerado o teste Binomial. Já para avaliação da acurácia dos diagnósticos citológicos entre as duas revisóes, foi estimada a estatística de kappa (к), que considera: concordância insignificante para valores menores que 0 ; fraca entre 0 e 0,2 ; razoável entre 0,21 e 0,4; moderada entre 0,41 e 0,6 ; forte entre 0,61 e 0,8 ; e quase perfeita entre 0,81 e 1 . Os intervalos de confiança (IC) de $95 \%$ foram utilizados e valores de $\mathrm{p}<0,05$ foram considerados estatisticamente significantes. Os dados foram analisados com o programa computacional Stata/ SE v.14.1. StataCorpLP, USA.

\section{RESULTADOS}

Durante janeiro de 2007 a dezembro de 2015, 21.041 lâminas de citologia cervical uterina foram avaliadas no SAP do CHC-UFPR, das quais $0,86 \%$ (181) se apresentaram com ASC-H e 1,39\% (293), com LIEAG.

Durante a busca no banco de dados do serviço, as 181 lâminas com ASC-H e as 293 com LIEAG foram diagnosticadas pelo mesmo patologista - esse dado foi elencado como importante para que a avaliaçáo de um único profissional fosse retestada pelo presente estudo. Dessas 474 amostras, 107 não apresentavam registro colposcópico no banco de dados, sendo, logo, excluídas do processo. Para que as amostras fossem reavaliadas, também foi necessário identificar seu resultado colposcópico durante o seguimento das pacientes, resultado este apontado como o desfecho diagnóstico. Das 367 restantes, 175 tiveram a avaliação colposcópica feita sem supervisão do colposcopista responsável pelo serviço. Dos 192 esfregaços remanescentes, somente 172 foram revisados, visto que 20 lâminas não foram encontradas no serviço com a mesma numeração do banco de dados da patologia cervical ou estavam inadequadas para avaliaçấo (Figura 1).

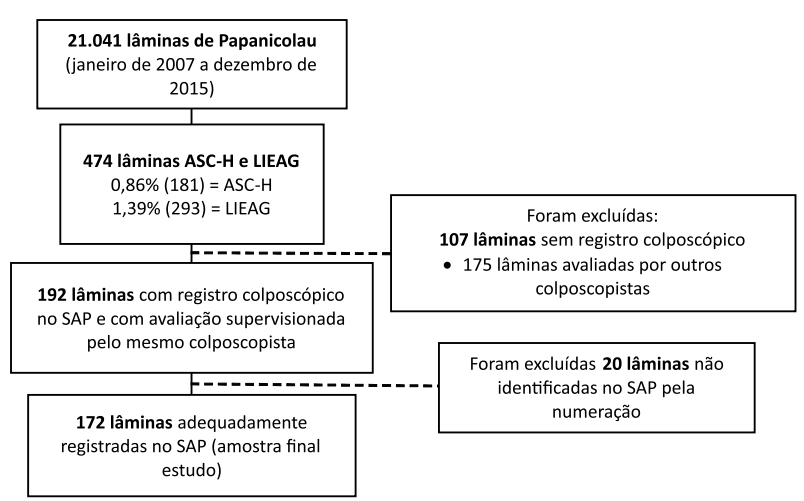

Figura 1. Fluxograma de obtenção das amostras do estudo

Legendas: ASC-H = células escamosas atípicas de significado indeterminado quando não se pode excluir lesão de alto grau; LIEAG = lesão intraepitelial escamosa de alto grau; SAP = Serviço de Anatomia Patológica.

As 172 lâminas selecionadas são referentes a 163 pacientes, uma vez que nove lâminas constituem repetição de exame citológico de nove pacientes que foram 
acompanhadas de acordo com o seguimento recomendado pelo Ministério da Saúde e aplicado pelo hospital.

Dos 172 esfregaços analisados no estudo, a prevalência de ASC-H na avaliação original é de 65,1\% (112 esfregaços), enquanto a de LIEAG, 34,9\% (60 esfregaços).

$\mathrm{Na}$ análise da concordância entre os resultados da avaliação original e aqueles elencados pela primeira revisão (Tabela 1), 61,6\% deles foram concordantes (IC 95\%; [54,4\%-68,9\%]).

Entre esses dados concordantes para a primeira revisão, a concordância para ASC-H é de 72,32\% e a de LIEAG, 41,67\%. Entre os diagnósticos ASC-H da avaliação original, em 22,32\% dos casos, o primeiro revisor interpretou como lesóes de menor grau de malignidade (negativo = 4,46\%; ASC-US = 16,07\%; e LIEBG $=1,79 \%$ ) e em 3,57\% como lesóes de alto grau. Entre os diagnósticos originais de ASC-H, 1,79\% deles foi interpretado como AG-US, assim como 1,67\% dos originalmente elencados, como LIEAG (Tabela 2).
Em relação à comparação dos resultados obtidos pela avaliação original e pela segunda revisão (Tabela 1), a concordância diagnóstica foi de 70,3\% (IC 95\%; [63,5\%- 77,2\%]).

A concordância para ASC-H, entre os diagnósticos concordantes da segunda revisão com a avaliação original, foi de $73,2 \%$, ao passo que para LIEAG, 65\%. Ademais, apenas $10,8 \%$ das lâminas ASC-H da avaliação original foram interpretadas como lesóes de menor grau (negativo $=4,5 \%$; ASC-US = 6,3\%) e 16,1\% dessas amostras foram tidas como lesóes de alto grau. Enquanto isso, os diagnósticos originais de LIEAG poderiam predizer lesóes de maior grau em $20 \%$ das ocasióes, enquanto $15 \%$ seriam, na verdade, lesóes de menor grau (negativo = 5\%; ASC-US $=5 \%$; LIEBG $=5 \%$ ). Nenhuma amostra fora classificada como AG-US após essa reescrutinação (Tabela 2).

As duas revisóes concordam com o diagnóstico original em 54,6\% dos casos e discordam, as duas, em 22,7\%. Além disso, em $7 \%$ das situaçóes, somente a primeira

Tabela 1. Concordância entre as revisões e a avaliação original

\begin{tabular}{ccccc}
\hline Concordância & \multicolumn{2}{c}{ Primeira revisão } & \multicolumn{2}{c}{ Segunda revisão } \\
\cline { 2 - 5 } & $\begin{array}{c}\text { Quantidade } \\
\text { absoluta }\end{array}$ & $\begin{array}{c}\text { Quantidade } \\
\text { relativa }\end{array}$ & $\begin{array}{c}\text { Quantidade } \\
\text { absoluta }\end{array}$ & Quantidade relativa \\
\hline Não & 66 & $38,4 \%$ & 51 & $29,7 \%$ \\
\hline Sim & 106 & $61,6 \%$ & 121 & $70,3 \%$ \\
\hline Total & 172 & $100 \%$ & 712 & $100 \%$ \\
\hline
\end{tabular}

Tabela 2. Comparação entre os resultados das revisões e da avaliação original

\begin{tabular}{|c|c|c|c|c|c|}
\hline \multirow{3}{*}{\multicolumn{2}{|c|}{$\begin{array}{c}\text { Distribuição dos resultados } \\
\text { das revisões }\end{array}$}} & \multicolumn{4}{|c|}{ Distribuição dos resultados da avaliação original } \\
\hline & & \multicolumn{2}{|c|}{ ASC-H } & \multicolumn{2}{|c|}{ LIEAG } \\
\hline & & $\begin{array}{c}\text { Quantidade } \\
\text { absoluta }\end{array}$ & $\begin{array}{c}\text { Quantidade } \\
\text { relativa }\end{array}$ & $\begin{array}{c}\text { Quantidade } \\
\text { absoluta }\end{array}$ & $\begin{array}{c}\text { Quantidade } \\
\text { relativa }\end{array}$ \\
\hline \multirow{7}{*}{ Primeira revisão } & $(-)$ & 5 & $4,46 \%$ & 0 & $0,00 \%$ \\
\hline & AG-US & 2 & $1,79 \%$ & 1 & $1,67 \%$ \\
\hline & ASC-H & 81 & $72,32 \%$ & 30 & $50,00 \%$ \\
\hline & ASC-US & 18 & $16,07 \%$ & 2 & $3,33 \%$ \\
\hline & LIEAG & 4 & $3,57 \%$ & 25 & $41,67 \%$ \\
\hline & LIEBG & 2 & $1,79 \%$ & 2 & $3,33 \%$ \\
\hline & Total & 112 & $100,00 \%$ & 60 & $100,00 \%$ \\
\hline \multirow{6}{*}{ Segunda revisão } & $(-)$ & 5 & $4,50 \%$ & 3 & $5,00 \%$ \\
\hline & ASC-H & 82 & $73,20 \%$ & 12 & $20,00 \%$ \\
\hline & ASC-US & 7 & $6,30 \%$ & 3 & $5,00 \%$ \\
\hline & LIEAG & 18 & $16,10 \%$ & 39 & $65,00 \%$ \\
\hline & LIEBG & 0 & $0,00 \%$ & 3 & $5,00 \%$ \\
\hline & Total & 112 & $100,00 \%$ & 60 & $100,00 \%$ \\
\hline
\end{tabular}

Legendas: $(-)$ = negativo; AG-US = células glandulares atípicas de significado indeterminado; ASC-US = células escamosas atípicas de significado indeterminado; ASC-H = células escamosas atípicas de significado indeterminado quando não se pode excluir lesão de alto grau; LIEBG = lesão intraepitelial escamosa de baixo grau; LIEAG = lesão intraepitelial escamosa de alto grau. 
revisão concordou com o diagnóstico original, enquanto, em $15,7 \%$ delas, apenas a segunda revisão concordou $(\mathrm{p}=0,024)$.

A concordância entre os revisores é considerada moderada, com valor de $\kappa$ igual a 0,46 (IC 95\%; [0,36 - 0,56]). Os diagnósticos que obtiveram as maiores concordâncias dentro dos $68,6 \%$ de diagnósticos concordantes foram ASC-H e LIEAG (81 e 27 lâminas, respectivamente). Por outro lado, o segundo revisor interpretou como lesóes de alto grau 25 das 111 lâminas classificadas como ASC-H pelo primeiro revisor. Somente cinco das 140 lâminas com diagnóstico de maior grau (ASC-H e LIEAG) pelo primeiro revisor foram classificadas como de menor grau pelo segundo (negativo = 2; $\mathrm{ASC}-\mathrm{US}=2$; e LIEBG = 1) (Tabela 3). O $\kappa$ específico para ASC-H e LIEAG foram, respectivamente, 0,49 e 0,52 - concordâncias moderadas.

Das 151 lâminas com lesôes de maior grau (ASC-H e LIEAG), de acordo com a segunda revisão, somente 13 foram interpretadas como de menor grau (negativo = 1; ASC-US = 10; e LIEBG = 2) pela primeira. E das 94 amostras com ASC-H tidas pela análise na segunda revisão, apenas duas foram entendidas como LIEAG pela primeira (Tabela 3).

Entre as 172 lâminas deste estudo, 142 delas foram interpretadas como ASC-H em alguma das três avaliaçôes. A análise do Desfecho dessas amostras, confirmada por meio do exame histológico, evidenciou que 68,3\% (97/142) dos casos de ASC-H corresponderam a lesôes de maior grau de malignidade (LIEAG $=61,3 \%$; CEC microinvasor $=1,4 \%$; e carcinoma escamocelular invasor $=5,6 \%$ ). Em contrapartida, 23,2\% das pacientes apresentaram resultado negativo a partir do exame padrão-ouro (Tabela 4).

Ainda nesse contexto, 6,3\% das pacientes não fizeram o exame padráo-ouro - 2,8\% porque não seguiram o acompanhamento orientado pelo médico, e 3,5\% em razão da repetição de outro exame citológico revelar uma lesão de menor grau de malignidade (ASC-US) (Tabela 4).

Por fim, a idade média das pacientes diagnosticadas com ASC-H deste estudo foi de 40,7 anos ( $\pm 13,5)$, variando entre 19 e 84 anos.

Tabela 4. Distribuição dos resultados quanto ao desfecho

\begin{tabular}{ccc}
\hline Desfecho & $\begin{array}{c}\text { Quantidade } \\
\text { absoluta }\end{array}$ & $\begin{array}{c}\text { Quantidade } \\
\text { relativa }\end{array}$ \\
\hline Perda de seguimento & 4 & $2,8 \%$ \\
\hline$(-)$ & 33 & $23,2 \%$ \\
\hline ASC-US & 5 & $3,5 \%$ \\
\hline LIEBG & 3 & $2,1 \%$ \\
\hline LIEAG & 87 & $61,3 \%$ \\
\hline CEC microinvasor & 2 & $1,4 \%$ \\
\hline CEC invasor & 8 & $5,6 \%$ \\
\hline Total & 142 & $100,0 \%$ \\
\hline
\end{tabular}

Legendas: $(-)$ = negativo; ASC-US = células escamosas atípicas de significado indeterminado; LIEBG = lesão intraepitelial escamosa de baixo grau; LIEAG = lesão intraepitelial escamosa de alto grau; CEC microinvasor = carcinoma escamocelular microinvasor; CEC invasor = carcinoma escamocelular invasor.

\section{DISCUSSÃO}

A nova terminologia de 2014 do Sistema de Bethesda definiu três princípios fundamentais, segundo os quais a terminologia deve comunicar informaçóes clinicamente relevantes a partir do laboratório para o médico responsável pelo atendimento das pacientes; ser uniforme e razoavelmente reprodutível entre diferentes patologistas e laboratórios, além de flexível o bastante para se adaptar a variados contextos laboratoriais e geográficos; e, por último, refletir a compreensão mais atual da neoplasia cervical ${ }^{8}$.

Tabela 3. Distribuição da concordância absoluta entre a primeira e a segunda revisão

\begin{tabular}{ccccccccc}
\hline $\begin{array}{c}\text { Distribuição absoluta da } \\
\text { segunda revisão }\end{array}$ & $\mathbf{7}$ & \multicolumn{7}{c}{ Distribuição absoluta da primeira revisão } \\
\hline AG-US & ASC-US & ASC-H & LIEBG & LIEAG & Total \\
\hline AG-US & 2 & 0 & 4 & 2 & 0 & 0 & 8 \\
\hline ASC-US & 0 & 0 & 0 & 0 & 0 & 0 & 0 \\
\hline ASC-H & 2 & 0 & 6 & 2 & 0 & 0 & 10 \\
\hline LIEBG & 0 & 2 & 9 & 81 & 0 & 2 & 94 \\
\hline LIEAG & 0 & 0 & 0 & 1 & 2 & 0 & 3 \\
\hline Total & 1 & 1 & 1 & 25 & 2 & 27 & 57 \\
\hline & 5 & 3 & 20 & 111 & 4 & 29 & 172 \\
\hline
\end{tabular}

Legendas: $(-)$ = negativo; AG-US = células glandulares atípicas de significado indeterminado; ASC-US = células escamosas atípicas de significado indeterminado; ASC-H = células escamosas atípicas de significado indeterminado quando não se pode excluir lesão de alto grau; LIEBG = lesão intraepitelial escamosa de baixo grau; LIEAG = lesão intraepitelial escamosa de alto grau.

Nota: Em cinza, encontram-se as quantidades absolutas de lâminas que obtiveram concordância diagnóstica após a revisão dos dois observadores. 
A prevalência de ASC-H e LIEAG no Brasil é, respectivamente, em torno de $0,2 \%$ e $0,26 \%$, entre todos os exames realizados, e de $8,8 \%$ e $9,1 \%$, levando em conta somente os alterados ${ }^{3}$. No presente estudo, a prevalência maior de diagnóstico limítrofe $(0,86 \%)$ e de LIEAG $(1,39 \%)$ entre todos os exames feitos pode ser explicada pelo fato de o Ambulatório de Tocoginecologia do CHC-UFPR ser referência para tratamento de doenças do trato genital inferior e receber pacientes previamente encaminhadas pelas UBS com exames previamente alterados.

Com predominância de esfregaços limítrofes $(65,1 \%$ de ASC-H versus 34,9\% de LIEAG), este estudo propôs avaliar o grau de concordância diagnóstica entre médicos-patologistas no CHC-UFPR para averiguar a reprodutibilidade técnica entre eles. A concordância entre a avaliação original e a primeira revisão foi de $61,6 \%$, ligeiramente menor do que sua unanimidade com a segunda revisão $(70,3 \%)$ e do que a consonância diagnóstica entre as revisôes $(68,6 \%)$.

Além disso, a primeira revisão apresentou maior congruência com os diagnósticos originais de ASC-H do que com os de LIEAG ( $72,32 \%$ versus $41,67 \%$, respectivamente), mas também levantou uma alta taxa de suspeita de malignidade para esses últimos resultados, classificando 50\% deles como ASC-H, evidenciando uma postura mais conservadora do patologista. A segunda revisão, por sua vez, mostrou concordância diagnóstica com as interpretaçôes originais de ASC-H quase semelhante à primeira $(73,2 \%)$. No entanto, também demonstrou maior concordância com os esfregaços originalmente laudados como LIEAG (65\%), se comparada à primeira, e identificou $20 \%$ destes como ASC-H, mostrando que essa patologista adotara uma postura mais arrojada no diagnóstico citológico. Na comparação dos resultados dos três avaliadores, foi encontrada evidência de que as duas revisóes diferem quanto à probabilidade de acerto com a avaliação original $(\mathrm{p}=0,024)$, ambas concordando somente em quase metade dos casos $(54,6 \%)$.

Esses dados apontam que, mesmo com uma discordância comprovadamente esperada no presente estudo, os resultados das revisōes tendem a classificar a maioria das lâminas como lesôes de maior grau de malignidade (ASC-H e LIEAG). Isso pode ilustrar o que foi recentemente abordado por Scheck et al. ${ }^{9}$, segundo os quais, quando há discordância entre citologia e histologia, pelo menos um resultado de maior grau de malignidade exige maior investigação, ou mesmo, reavaliação. Nesse estudo, o seguimento durante cinco anos de pacientes com diagnóstico citológico de ASC-H e LIEAG mostrou 30\% a 50\% de chance de evolução para lesóes malignas.
Nesse ponto, as concordâncias para ASC-H entre os revisores e o laudo citológico original das pacientes mostraram-se maiores do que a apontada pelo estudo BIRST-2 $(60 \%)^{7}$. Esse achado corrobora a tendência encontrada pelos autores em relação ao BIRST $^{6}$ de que os diagnósticos limítrofes têm incrementado seu grau de consonância diagnóstica interobservador. Para alguns autores $^{5}$, a experiência do observador constitui-se como fator importante para o diagnóstico citológico adequado. Outros $^{10}$ elencam demais possíveis determinantes para a variabilidade dos resultados: a sobrecarga de trabalho, a dificuldade diagnóstica inerente à categoria de atipias indeterminadas, sendo, portanto, extremamente necessário o bom relacionamento entre o ginecologista $\mathrm{e}$ o citopatologista na busca pelo exame de qualidade.

Existe evidência ${ }^{8}$ de que as características morfológicas dos esfregaços são mais determinantes do que o grau acadêmico ou profissional dos observadores (proeminência nucleolar cercada por neutrófilos e núcleos aumentados com brandas apresentaçôes de cromatinas - achados representativos de reparo tecidual que foram elencados como aspectos de lesóes de malignidade). Outros autores ${ }^{11}$ apontam que sessôes continuadas envolvendo a discussão de casos discordantes, a revisão de esfregaços e a padronização de critérios citopatológicos influenciam bastante no grau de discordância interobservador.

Assim como os achados deste estudo, outros autores com resultados semelhantes atribuem ao Sistema de Bethesda as boas taxas de reprodutibilidade diagnóstica ${ }^{12}$, afirmando que os critérios do Sistema de Bethesda podem ser assimilados e rotineiramente aplicados. E isso vai ao encontro do princípio da flexibilidade da terminologia, a qual deve se adaptar a diversos contextos técnicos e regionais.

Ainda neste estudo, a concordância diagnóstica entre os dois revisores foi considerada moderada $(\kappa=0,46)$, demonstrando boa reprodutibilidade entre eles. Outros estudos também apresentam concordância moderada entre seus avaliadores ${ }^{7,12}$, mas também apresentam a categoria ASC-H como a de pior reprodutibilidade de seus estudos ( $\kappa$ específico entre 0,19 e 0,38 - concordâncias baixa a razoável) $)^{5}$. Esses dados sugerem que a terminologia ASC-H ainda possui reduzida reprodutibilidade entre as demais categorias diagnósticas desse Sistema.

Nesta pesquisa, entretanto, os $\kappa$ específicos para ASC-H e LIEAG náo foram tão distintos e foram considerados de moderada concordância $(0,49$ e 0,52 , respectivamente). A explicação para a elevação da reprodutibilidade para ASC-H e a diminuição dessa para LIEAG deve-se ao viés de seleção dos casos elencados. O estudo levou em consideração apenas esfregaços originalmente diagnosticados como ASC-H ou LIEAG (havendo algum diagnóstico citológico limítrofe no seguimento desses 
últimos casos), o que pode náo representar uma amostra confiável da realidade. Igualmente, também ocorreu o viés de aferição na revisão dos patologistas, uma vez que eles sabiam qual era o objetivo do estudo e quais seriam possivelmente as lâminas que estariam estudando.

A frequência de LIEAG foi de $61,3 \%$ e a de carcinoma $7 \%$ nas mulheres com citologia de ASC-H. Essa prevalência encontra-se dentro das margens de $12,2 \%$ a 68\% (para LIEAG) e de 1,3\% a 39\% (para o carcinoma cervical) esperadas pela literatura ${ }^{3}$. Um ponto importante desta pesquisa é que a taxa de mulheres que não seguiram o protocolo preconizado pelo Ministério da Saúde foi baixa $(2,8 \%)$ em comparação aos dados atuais. Conforme a literatura nacional mais recente ${ }^{13}$, após o diagnóstico de ASC-H, nenhuma conduta foi adotada em $40,5 \%$ das mulheres atendidas no Estado do Ceará. O CHC-UFPR é um hospital universitário cuja missão é garantir um ambiente apropriado ao ensino, à pesquisa e à extensão, possuindo em seu corpo clínico médicos e professores com grande experiência e formação. Isso pode ser visto como um potencial determinante na adequada adesão ao seguimento das pacientes frente ao resultado ASC-H do presente estudo.

De encontro ao que preconiza a Organização Mundial da Saúde, o padrão predominante do rastreamento no Brasil é oportunístico, isto é, as pacientes têm realizado o exame citopatológico quando buscam os serviços de saúde por outras razóes. Em consequência disso, aproximadamente $21 \%$ dos exames têm sido feitos fora da faixa etária recomendada ${ }^{14}$. Com isso, existe um grupo de mulheres rastreadas acima do recomendado e outro grupo sem qualquer exame de rastreamento, comprometendo $\mathrm{o}$ acesso daquelas que realmente precisam ser triadas e encaminhadas para a investigação diagnóstica e tratamento das lesôes precursoras. Na presente análise, $0,08 \%$ das pacientes tinham menos do que 25 anos, mas isso pode ser um dado subestimado, posto que somente lâminas com duas categorias foram analisadas.

A idade média das pacientes diagnosticadas com ASC-H deste estudo foi de 40,7 anos $( \pm 13,5)$, variando entre 19 e 84 anos. Houve, nesse sentido, pacientes que foram triadas antes dos 25 anos (ponto de partida da triagem do carcinoma do colo uterino). Apesar de existir evidência ${ }^{15,16}$ de maior probabilidade de regressão de lesōes pré-invasivas até os 24 anos de idade, essas pacientes foram conduzidas pelo serviço do hospital de acordo com as Diretrizes do Ministério da Saúde ${ }^{3}$.

Uma importante limitaçáo deste estudo refere-se ao fato de que os revisores sabiam que o objetivo do estudo era avaliar o grau de concordância interobservador para a categoria diagnóstica de ASC-H do Sistema de Bethesda. Isso pode ter induzido, por sua vez, os patologistas a interpretarem os achados como lesóes de maior grau de malignidade e também pode ter contribuído para a menor concordância de LIEAG em relação ao apontado pela comunidade científica. Outro limitante do estudo foram as amostras não incluídas em virtude dos fatores de exclusão eleitos. A ausência desses esfregaços pode ter influenciado a epidemiologia do presente estudo, mas é importante reforçar que havia barreiras físicas e diagnósticas que impossibilitaram sua consideração nessa pesquisa.

Apesar das limitaçóes anteriormente comentadas, este estudo contribuiu para a conscientizaçấo da necessidade de educação continuada em citologia cervical para os patologistas do CHC-UFPR. Ênfase deve ser dada à abordagem de lesóes de significado indeterminado, que foi a categoria diagnóstica de maior discordância entre os patologistas revisores (Tabela 3), em que nove diagnósticos de ASC-US da primeira revisão corresponderam a ASC-H na segunda. Essa discordância tem como consequência diferentes condutas clínicas e deve ser abordada exaustivamente na educação continuada, assim como as lesóes intraepiteliais de alto grau, para que esses profissionais uniformizem seus critérios morfológicos.

\section{CONCLUSÃO}

Houve moderada concordância interobservador incluindo os diagnósticos de ASC-H e LIEAG. Assim também, a concordância específica para ASC-H foi moderada. O diagnóstico de ASC-H correspondeu à lesão de maior grau de malignidade na histologia, o que demonstra que essas lesóes devem ser seguidas como LIEAG.

Há necessidade de educação continuada em citologia cervical dos patologistas do CHC-UFPR para uniformizar os critérios diagnósticos de ASC-H e LIEAG de acordo com o Sistema de Bethesda.

\section{CONTRIBUIÇÕES}

Todos os autores contribuíram substancialmente na concepção e/ou planejamento do estudo; na obtenção, análise e/ou interpretaçáo dos dados; na redação e revisão crítica; e aprovaram a versão final a ser publicada.

\section{DECLARAÇÃO DE CONFLITO DE INTERESSES}

Nada a declarar.

\section{FONTES DE FINANCIAMENTO}

Não há. 


\section{REFERÊNCIAS}

1. Instituto Nacional de Câncer José Alencar Gomes da Silva [Internet]. Rio de Janeiro: INCA; [data desconhecida]. Tipos de câncer: câncer do colo do útero; [modificado 2021 nov 18; acesso 2021 abr 22]. Disponível em: https://www.inca.gov.br/tipos-de-cancer/cancer-docolo-do-utero

2. Ellenson LH, Pirog EC. O trato genital feminino: colo uterino. In: Kumar V, Abbas AK, Aster JC, editores. Robbins \& Cotran: patologia: bases patológicas das doenças. 9. ed. Rio de Janeiro: Elsevier; c2016. p. 1848-57.

3. Instituto Nacional de Câncer José Alencar Gomes da Silva. Diretrizes brasileiras para o rastreamento do câncer do colo do útero. 2. ed. rev. ampl. atual. Rio de Janeiro: INCA; 2016.

4. Baena A, Guevara E, Almonte M, et al. Factors related to inter-observer reproducibility of conventional Pap smear cytology: a multilevel analysis of smear and laboratory characteristics. Cytopathology. 2017;28(3):192-202. doi: https://doi.org/10.1111/cyt.12410

5. Confortini M, Di Stefano C, Biggeri A, et al. Daily peer review of abnormal cervical smears in the assessment of individual practice as an additional method of internal quality control. Cytopathology. 2016;27(1):35-42. doi: https://doi.org/10.1111/cyt.12195

6. Sherman ME, Dasgupta A, Schiffman M, et al. The Bethesda Interobserver Reproducibility Study (BIRST): a web-based assessment of the Bethesda 2001 system for classifying cervical cytology. Cancer. 2007;111(1):15-25. doi: https://doi.org/10.1002/cncr.22423

7. Kurtycz DFI, Staats PN, Chute DJ, et al. Bethesda Interobserver Reproducibility Study-2 (BIRST-2): Bethesda system 2014. J Am Soc Cytopathol. 2017;6(4):131-44. doi: https://doi.org/10.1016/j. jasc.2017.03.003

8. Sebastião APM, Noronha L, Scheffel DLH, et al. Estudo das atipias indeterminadas em relação à prevalência e ao percentual de discordância nos casos do Programa de Prevenção do Câncer Uterino do Paraná. J Bras Patol Med Lab. 2004;40(6):431-8. doi: https://doi.org/10.1590/ S1676-24442004000600012

9. Scheck SM, Liddle C, Wood Z, et al. Five-year followup after cervical cytology and histology discordance: a retrospective cohort study. Aust N Z J Obstet Gynaecol. 2021;61(3):424-9. doi: https://doi.org/10.1111/ ajo. 13329

10. Davey DD. Bethesda cervical cytology interobserver reproducibility: have we reached the sweet spot? J Am
Soc Cytopathol. 2017;6(4):127-30. doi: https://doi. org/10.1016/j.jasc.2017.05.002

11. Morais LSF, Magalhães JC, Braga IS, et al. Performance of laboratories after 10 years of participating in external quality monitoring in cervical cytology. Acta Cytol. 2020;64(3):224-31. doi: https://doi. org/10.1159/000502433

12. Sørbye SW, Suhrke P, Reva BW, et al. Accuracy of cervical cytology: comparison of diagnoses of 100 Pap smears read by four pathologists at three hospitals in Norway. BMC Clin Pathol. 2017;17:18. doi: https://doi.org/10.1186/ s12907-017-0058-8

13. Oliveira GG, Oliveira JMSC, Eleutério RMN, et al. Management of atypical squamous cell cases: a prospective study of women seen at a private health service in Northeastern Brazil. Rev Bras Ginecol Obstet. 2018;40(3):121-6. doi: https://doi. org/10.1055/s-0038-1639466

14. Instituto Nacional de Câncer José Alencar Gomes da Silva. Monitoramento das açóes de controle dos cânceres de mama e de colo uterino. Informativo Detecção Precoce [Internet]. 2018 jan-jun [acesso 2021 abr 22];9(1):18. Disponível em: https://www.inca.gov.br/sites/ufu. sti.inca.local/files//media/document//informativo_ deteccao_precoce_numero1_2018.pdf

15. Moscicki AB. Management of adolescents who have abnormal cytology and histology. Obstet Gynecol Clin North Am. 2008;35(4):633-43;x. doi: https://doi. org/10.1016/j.ogc.2008.09.004

16. McAllum B, Sykes PHH, Sadler L, et al. Is the treatment of CIN 2 always necessary in women under 25 years old? Am J Obstet Gynecol. 2011;205(5):478.e1-47.e7. doi: https://doi.org/10.1016/j.ajog.2011.069. 